\title{
Process integrated production of wc-co tools with local cobalt gradient fabricated by binder jetting
}

\author{
Maja Lehmann, Michael F. Zaeh \\ Institute for Machine Tools and Industrial Management (iwb), Department of Mechanical Engi- \\ neering, Technical University of Munich, Boltzmannstr. 15, 85748 Garching, Germany
}

\begin{abstract}
Producing complex shaped tungsten carbide cobalt (WC-Co) tools by classical technologies is difficult and often impossible due to their high hardness and brittle fracture behavior. Additive Manufacturing (AM) is a suitable technology for creating complex structures and simultaneously shortening expensive machining processes. Binder Jetting (BJ) is an innovative AM technology that offers several advantages over laser-based processes, for example low manufacturing costs and high build-up rates. Binders with nanoparticle additives have already proven to be effective in increasing the packing density of the powder bed and improving the sintering properties. Additionally, they offer the possibility of selectively changing the material composition in the part. This paper presents a concept for the use of nanoparticles to generate gradients in the green compact, which leads to a cobalt gradient in the part after sintering. The possibility of introducing particles locally into complex structures allows local modification of the material properties.
\end{abstract}

\section{Keywords:}

Additive Manufacturing, Binder Jetting

\section{Introduction}

Due to their high hardness, superior wear resistance and acceptable toughness, tungsten carbide cobalt (WC-Co) tools are primarily used for turning, milling or drilling. However, during these machining processes, high temperatures reduce the cutting ability and decrease the wear resistance of the tool so that a cooling lubricant is required. Integrated cooling channels in the tool are essential to ensure that the cooling lubricant can be fed into the process zone as required. Owing to the very high hardness and brittle fracture behavior of hard metals, only a few manufacturing technologies are suitable for processing them. Until now it has only been possible to produce complex integrated cooling channels at very high cost. AM offers a simple and cost-effective alternative: the layer-by-layer generation of parts enables the production of complex structures, which cannot be fabricated by conventional powder metallurgical manufacturing methods. Near-netshape production reduces or shortens complex machining processes, because less material has to be removed during post-processing and there is no need for tooling. This leads to more resourceefficient production $[1,2]$.

Binder Jetting is a promising additive manufacturing process for the fabrication of WC-Co parts. It has advantages over laser-based processes because of its low unit costs and high build-up rates. During BJ, a binder selectively bonds the powder layers to create a green part, which is then densified in a thermal process, similar to the powder metallurgical production of WC-Co parts. A disadvantage of BJ is the low green part density, which results in high finished part porosity. A suspension of binder and nanoparticles increases the packing density of the powder bed and improves the sintering properties [3], resulting in higher part density. The particles can also introduce specific composition changes in the part resulting in gradients that can improve the mechanical properties and wear resistance. 
The cobalt concentration determines the local properties of WC-Co parts [4]. In principle, fracture toughness and wear resistance exhibit opposing behavior: an increase in the cobalt content increases the toughness but decreases the hardness and hence the wear resistance [5, 6]. Creating a cobalt gradient in a tool can offer superior combinations of toughness and hardness, resulting in more durable tools and superior engineering performance [6]. For example, the cutting surface of a milling tool requires high hardness to minimize wear. However, other areas of the tool, such as the shank, are exposed to dynamic loads, consequently improved ductility is required to prevent fracture and reduce the risk of failure.

This paper presents a theoretical concept for introducing cobalt gradients in WC-Co tools and thereby for tailoring their mechanical properties. Currently, cobalt gradients are generated either by surface technologies [7] or in a layer-by-layer mode [8] and are thus limited to the outer skin or defined but complete layers. Selectively setting the cobalt concentration anywhere in the component is not yet possible. This work illustrates an approach for overcoming this shortcoming: as key $\mathrm{BJ}$ is employed to produce location-independent voxel-based cobalt gradients during green part production.

\section{BJ of WC-Co parts}

\section{State of the Art}

To the knowledge of the authors, very few studies and research contributions are available on the BJ of WC-Co. The processability of WC-Co with 12 wt \% Co by BJ was demonstrated by [2]. Afterdensification by pressure sintering, samples with a density of $14.1 \mathrm{~g} / \mathrm{cm}^{3}$ could be manufactured. The pressure applied during sintering was only a fraction of the pressures usually employed in hot isostatic pressing. The mechanical properties of the sintered parts corresponded to the hardness and the toughness of conventional production with the same composition. However, the structure of the WC grains showed coarse grain growth, which had a negative effect on the mechanical properties. The infiltration of a sintered porous WC skeleton with pure cobalt was considered as an alternative for the manufacturing of WC-Co parts [9]. However, this method achieved high part densities at the expense of an additional process step, increased production time, and extra costs.

In summary, it can be stated that the production of dense WC-Co parts using BJ has been found to be possible, however, the mechanical properties obtained thus far are not yet comparable with those achieved from conventional production processes. Also, where dimensional accuracy and shrinkage effects are concerned, novel approaches need to be found to permit successful industrialization of the BJ of WC-Co.

\section{Functionally graded WC-Co parts}

Various approaches have been explored to create graded structures in WC-Co materials [4]. Many of these approaches are based on the physical effect of liquid phase migration. An established method for creating a cobalt gradient is the carburizing process [6]. The process consists of conventional liquid phase sintering followed by carburizing heat treatment. During the process, the temperature lies in the three-phase equilibrium range between solid WC, solid cobalt and liquid cobalt. The formation of the cobalt gradient during the carburizing heat treatment depends on two principles: the first principle relates to the volume fraction of the liquid cobalt in the three-phase region. This volume fraction depends on the carbon content. The proportion of the liquid cobalt rises with increasing carbon content at the expense of the solid cobalt. The second principle responsible for the formation of the gradient is the liquid phase migration of the cobalt from a cobaltrich region to a cobalt-poor region. 
Before the carburization begins, the carbon content as well as the cobalt content are evenly distributed over the entire volume of the part. Moreover, the prevailing temperature is approximately $1300^{\circ} \mathrm{C}$ and the resulting liquid cobalt is homogeneously distributed. When carburization begins, the carbon content increases on the surface of the part. Simultaneously, the proportion of liquid cobalt increases in that area. The higher proportion of liquid cobalt near the surface means an imbalance in the distribution of the liquid phase. This causes a driving force for the liquid phase migration of the cobalt to the middle of the part. As a result, a gradient from the surface to the core is formed [6].

Parts with independently controlled (e.g. voxel-based) WC-Co composition at different points have yet to be produced. A conceivable way of generating gradients within a part is the production of a graded powder compact, i.e. a powder compact with a graded cobalt composition. However, this gradient is quickly compensated during liquid phase sintering so that the finished part has a homogeneous structure. By contrast, the graded structure can be preserved during solid phase sintering. However, this sintering process usually results in a residual porosity in the part. Consequently, the application of pressure by means of hot isostatic pressing or spark plasma sintering is necessary to produce a dense part with a graduated structure [8]. However, industrial application of this manufacturing method is limited because of the additional step and increased planning and operating costs [6].

One approach to the formation of graded structures in WC-Co parts and the simultaneous achievement of a high part density is control of the liquid phase migration of cobalt using short-time liquid phase sintering. During short-time liquid phase sintering, the gradient can be maintained without the disadvantage of lower part density. The liquid phase migration and consequently the gradient can be controlled by three factors: the volume fraction of liquid cobalt, the carbon content, and the WC grain size. If one of these factors is applied, the gradient does not compensate during liquid phase sintering [6].

\section{Printing of nanoparticle loaded binders}

The production of complex geometries, the construction of parts without a building platform or support structures, and the high build-up rates are among the many advantages of BJ. Reducing the porosity of the parts is still one of the biggest challenges of the process. One way of reducing the porosity and increasing the density of the parts is the introduction of particles via the print head $[10,11]$. The nanoparticles improve the packing density of the powder bed and due to the high surface areas of the particles the sintering driving force is increased. Additionally, these particles can be used to introduce gradients or for multi-material processing, which can be realized by using nanoparticles of different materials [12].

The creation of tailor-made properties in steel parts using BJ is one example of multi-material processing. To do so, a binder mixed with carbon nanoparticles is printed on the steel-metal powder bed. With this technology, GODLINSKI ET AL. [12] produced graded green bodies, which were subsequently sintered. The elements in the binder acted as alloying elements and changed the local strength and hardness properties. However, according to GODLINSKI ET AL. [12] the main limitation was data preparation software which was not fully developed and did not support the production of tailor-made reproducible parts. The application of gradients could not be implemented in the software so that consequently automated production was not possible. Additionally, missing materials and process procedures caused difficulties [12].

Furthermore, nanoparticles can change the sintering process; B AI ET AL. [3] added silver nanoparticles to the binder and printed the suspension on a powder bed of silver microparticles to improve the properties of printed silver parts. The nanoparticles melt at lower temperatures than the larger 
particles and the sintering onset temperature is lower than the binder burn-out temperature. Thus, the consolidation of the smaller particles resulted in a higher bonding strength during the heating process before the binder burned out. This effect led to a higher green part strength, improved the dimensional accuracy, and increased the ductility of the silver parts [3].

The use of nanoparticles can also have a negative effect on the porosity: BAILEY ET AL. [11] observed that a high nanoparticle content in the binder increased the porosity during the sintering process forming large, round pores. The nanoparticles seemingly blocked the exit of the evaporating binder, leaving gas inclusions in the part [11]. Therefore, it is important to study the highest possible nanoparticle content in the binder to still profit from the positive effect on the density.

The properties of the binder change depend on the amount of particles. The surface tension of the binder decreases with increased particle content, resulting in a reduced penetration depth into the powder bed. In addition, the rheological properties change depending on the particle charge. The particle charge of the binder is therefore a limiting factor of the process in respect of the layer thickness [10].

\section{Approach and theoretical considerations}

\section{Concept}

The concept presented in this paper is based on the hypothesis that a process-integrated production of WC-Co tools with locally adapted cobalt gradients can be achieved by the integration of nanoparticles through BJ. Using nanoparticles is a common way to improve the density of the sintered part. During the printing process the small particles fill the voids between the particles of the powder bed, thus the packing factor of the powder feedstock is increased. This factor dictates the final density and the shrinkage of the part. The nanoparticles may also function as sintering aids, which also improves the final density [11].

In addition to these effects, generation of a cobalt gradient can be triggered by nanoparticles in the green part during the sinter process. Consequently, a nanoparticle gradient has first to be generated in the green part. Suspensions containing carbon or WC nanoparticles were selected as binders. To create the gradients, nanoparticles are selectively distributed in relation to the desired gradient positions. For this purpose, a system set-up with two print heads is used. One print head is able to print binders with additional nanoparticles and another print head prints pure binder (see Figure 1).

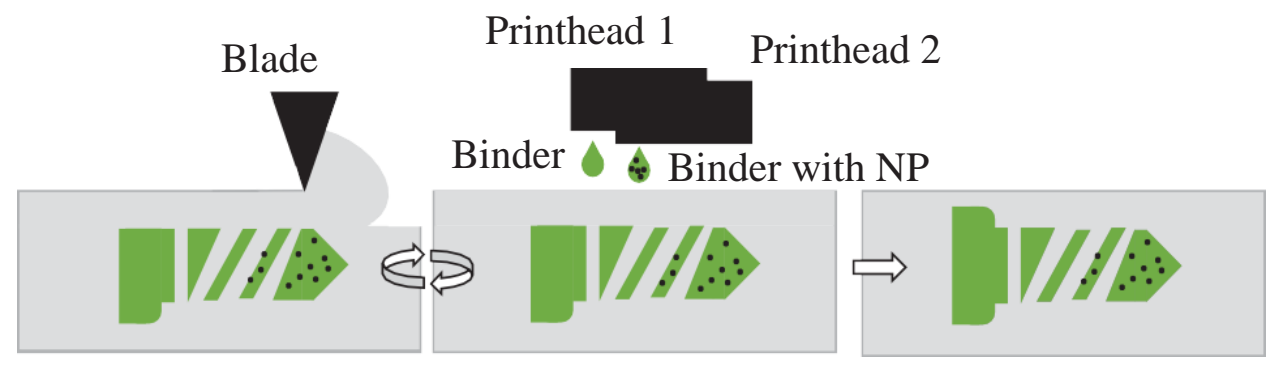

Finished green part

Figure 1: Schematic representation of the modified BJ process adapted from [13]

The generation of the cobalt gradient and the selection of WC and carbon as nanoparticles can be explained by considering the effects occurring during the liquid phase sintering process. The carbon content significantly influences the cobalt gradient. A high carbon content leads to a rise in liquid cobalt at the expense of solid cobalt creating a liquid cobalt gradient that causes a 
cobalt migration (see Figure 2) [6]. The printability of carbon-based binders for BJ has already been described in the literature and is feasible [12]. However, the introduction of too much carbon can result in the formation of a graphite phase, which is considered to be a defect in cemented carbide tools [1].

(1) less liquid-Co

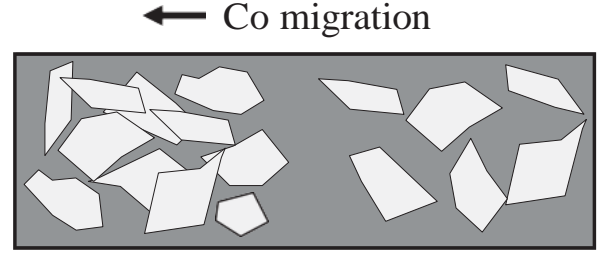

more liquid-Co

(2)

finer WC

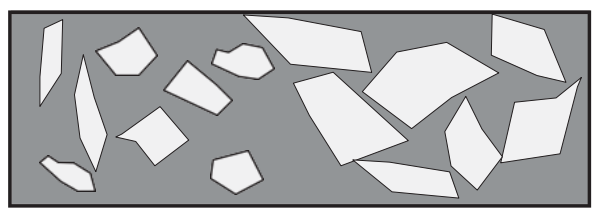

coarser WC

(3) lower C content

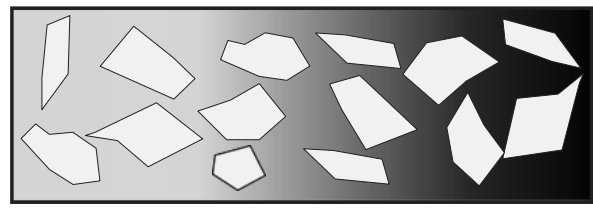

higher $\mathrm{C}$ content

Figure 2: Direction of cobalt migration depending on three different effects: 1) amount of liquid cobalt, 2) WC grain size and 3) carbon content adapted from [6]

Hence, a WC-based binder is investigated as an alternative. We anticipate that the WC particles trigger the formation of liquid cobalt and thus affect the gradient in a similar way to carbon (see Figure 2). Additionally, we expect this to be amplified by grain growth due to the effect of Ostwald ripening that is caused by the WC nanoparticles [14]: WC grains grow more strongly in the areas with a higher amount of nanoparticles than in other areas of the part. This also leads to cobalt migration. As shown in Figure 2, cobalt migration is increased by both coarser WC and higher carbon content. Hence, we assume that the creation of a cobalt gradient can be enabled by using WC nanoparticles to increase the WC grain size or by using carbon nanoparticles to increase the carbon content.

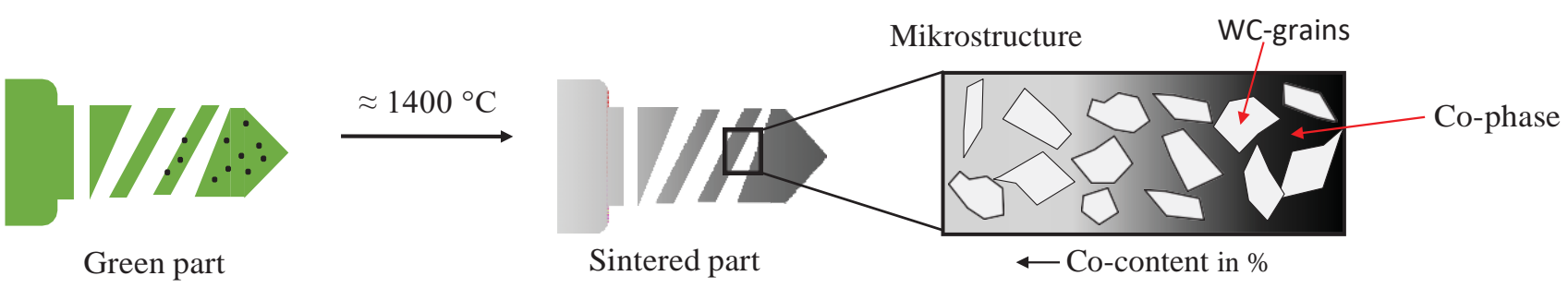

Figure 3: Schematic representation of the sintering process and microstructure formation 
The basic hypothesis of the presented concept is that the effect of cobalt migration can be used to convert the nanoparticle gradient in the green part into a cobalt gradient in the sintered part (see Figure 3). It is expected that during sintering cobalt migration occurs due to the triggering effect of the selected nanoparticles and a graded microstructure forms. The graded microstructure is responsible for the changed mechanical properties of the tool.

\section{Relevant input and output variables}

Different input variables influence the formation of the cobalt gradient, the resulting mixed phases and the porosity of the final part. The relevant input and output variables of the process are shown in Figure 4.

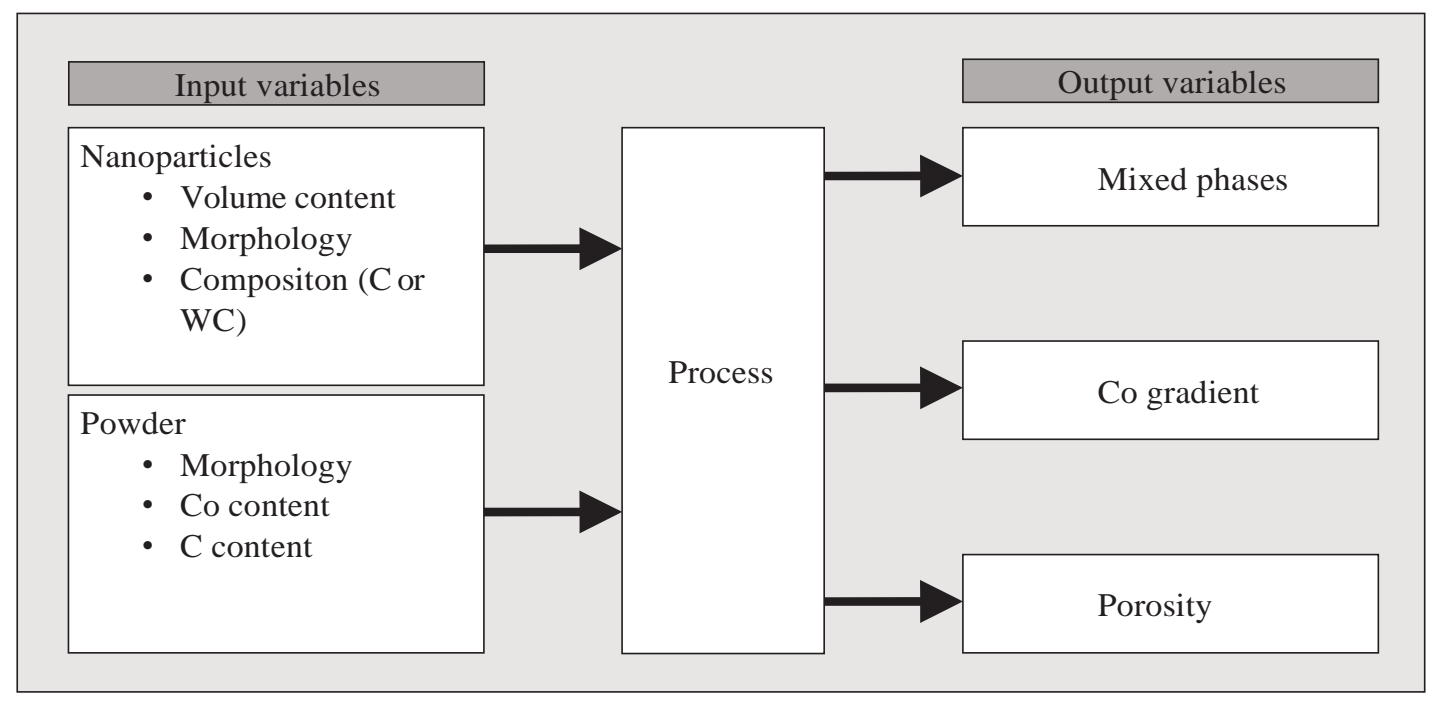

Figure 4: Input and output variables of the process

Where the nanoparticles are concerned, volume content, morphology, size, and composition are important for the process. The powder used is also an important factor for the process: the morphology, cobalt content, and carbon content influence all three aspects of the output variables. The morphology has a significant effect on the powder packing factor as well as on the flowability and thus on the porosity [15]. The cobalt content of the powder determines the toughness. If it is too low, no gradient can be generated. If it is too high, the liquid phase migration might be too strong to develop a gradient.

The carbon content of the powder has a notable influence on the formation of the mixed phases. A low carbon content results in the brittle Eta-phase, while a high carbon content leads to a graphite phase. Both of these phases are considered to be defects in tools [16, 17].

If residual carbon remains after the binder has burnt out, the carbon content in the powder needs to be adapted to avoid undesired phases. Powders with different morphologies and different carbon contents are to be tested for the process. The cobalt content is to be kept constant for the first tests. All input variables must be varied systematically to investigate the effect on the respective output variables. In this way the underlying cause-effect relationship can be understood and suitable methods identified for controlling the formation of the cobalt gradient. 


\section{Major challenges}

Printability of binders containing nanoparticles: various factors have an effect on the printability of particle-loaded binders. Reliable printing behavior is based on the liquid rheology parameters, being influenced by viscosity, surface tension, and the stability of the suspension [15]. All three aspects depend on the particles. To guarantee the generation of droplets, the surface tension and the viscosity must be adjusted to match the requirements of the print head. Excessive particle loads can negatively influence these properties. The suspension stability depends on the particle size, the agglomeration of particles, and the viscosity of the binder [15]. Since nanoparticles tend to agglomerate easily, a dispersant is needed to ensure a stable suspension. The stability is controlled by monitoring the sedimentation over time and by measuring the zeta-potential and the hydrodynamic diameter [18]. For the WC suspension in particular, fundamental investigations are required to determine the stability because $\mathrm{WC}$ has a high density and the authors could not find any related literature for the preparation of binders loaded with WC. In contrast to WC, the carbon has a lower density. Consequently the carbon suspension is expected to be stable with a suitable dispersant and without it being necessary to add further additives to the binder. Droplet formation is observed using a high speed camera and the mass of a certain number of droplets is measured to ensure reliable printing. High variability in mass indicates an unstable binder. The size of the agglomerates must be controlled in order to be able to print a binder loaded with nanoparticles. The most suitable type of print heads uses the drop-on-demand principle and has a nozzle diameter of $50 \mu \mathrm{m}$ : this means that the agglomerates need to be at least 50 times smaller to prevent clogging or bridging of the nozzle [15].

Control of cobalt gradient propagation: the cobalt gradient strongly depends on the amount of particles delivered into the powder bed. This amount can be controlled via the particle loading and binder saturation. The particle loading is controllable and measurable but the binder saturation depends on the interaction between binder and powder bed including the spreading and the penetration of the binder into the powder bed. For this interaction, MIYANAJI AND YANG developed a physics-based model to estimate the saturation level [19]. By adapting their results to a fluid with particle loading, the penetration of the particles is predictable. If the penetration depth of the nanoparticles can be predetermined, it is possible to correlate the influence and the location of the particles with the formation of the gradient. Using this information, a controlled local formation of the cobalt gradient is expected to be feasible.

\section{First results}

\section{Material and Methods}

The machine used for this investigation is a 3D printer type Voxeljet VTS128 (Voxeljet Technology $\mathrm{GmbH}$, Augsburg, Germany). It is a test rig with a process chamber with the dimensions (xyz) $250 \times 110 \times 95 \mathrm{~mm}^{3}$ and it is equipped with a combined powder feeder and a cylindrical recoater. The print head is a spectra SL-128 AA module (Fujifilm Dimatric Inc.) with 128 nozzles and a nozzle spacing of $0.508 \mathrm{~mm}$.

For the binder tungsten carbide powder (IoLiTec Ionic Liquids Technologies GmbH, Heilbronn, Germany) with particles smaller than $200 \mathrm{~nm}$ was mixed with an aqueous binder (Additive Elements GmbH, Planegg, Germany). To suspend the nanoparticles in the liquid, improve the stability of the suspension, and avoid agglomeration, different dispersion techniques were used. The suspension was mechanically stirred, an ultrasonic bath was used to disperse the particles and various dispersants with different concentrations were investigated. After a literature review, polyethylenimine (PEI, Sigma Aldrich) [21] and sodium polyphosphate (Graham's salt, Sigma Aldrich) [22] were chosen as dispersants. The dispersant Zetasperse 179 (Evonik) was also used as an alternative. 
The particle distribution was measured by laser light scattering (Mastersizer 3000E, Malvern Panalytical).

Two powders with different morphologies and various carbon contents were investigated and their suitability for BJ was analyzed. The aims were to determine if the flowability of the irregular particles differs greatly from that of spherical particles and if the particle density and the bulk density have an influence on the sinter density. Additionally, it was also investigated if the residual carbon of the binder is sufficient to compensate for the low carbon content of the powders. Table 1 gives a detailed description of the powders used in the first experiments.

Table 1: Chemical composition and bulk density of the two WC-Co powders used in this study

\begin{tabular}{cccccc} 
Powder & Supplier & $\begin{array}{c}\text { Particle size } \\
\text { in } \mu m\end{array}$ & $\begin{array}{c}\text { Bulk density } \\
\text { in } \mathrm{g} / \mathrm{cm}^{3}\end{array}$ & $\begin{array}{c}\text { Co content } \\
\text { in mass } \%\end{array}$ & $\begin{array}{c}\text { C content } \\
\text { in mass } \%\end{array}$ \\
\hline Amperit 515 & H.C. Starck & $45 / 22$ & 6.31 & 12 & $3.9-4.3$ \\
WC-793 & Praxair & $<53$ & 5.25 & 11 & 4.2
\end{tabular}

\section{Results and discussion}

Figure 5 (left) depicts Amperit powder. It has a blocky and irregular shape. By contrast, WC-793 (Figure 5 right) has a spherical shape but, due to the manufacturing method, the powder is highly porous has.
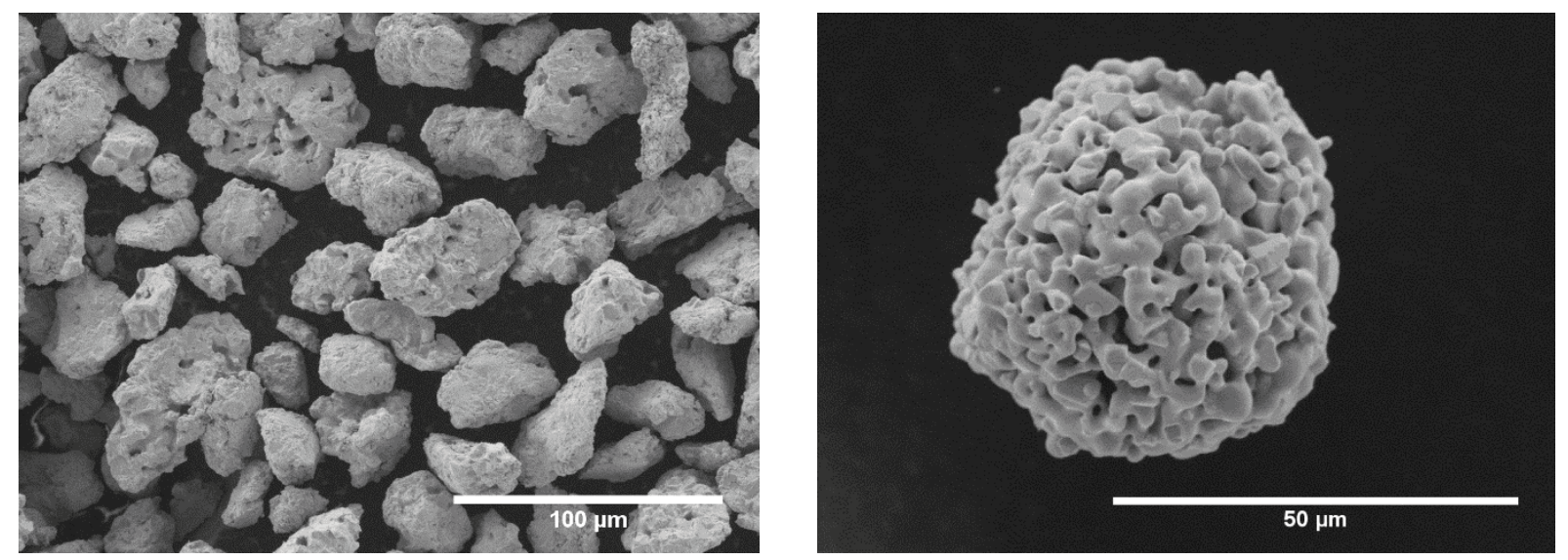

Figure 5: Morphology of the WC-Co powders: left Amperit 515; right WC-793

Although the flowability is influenced by the morphology, and spherical particles should have better flow properties [20], no significant difference was observed during powder deposition. Figure 6 shows the spread powder bed including the binder deposition. Material clumps or pits in the powder bed were not visible. The powder bed of both powders exhibited a smooth top surface after application of new layers. 


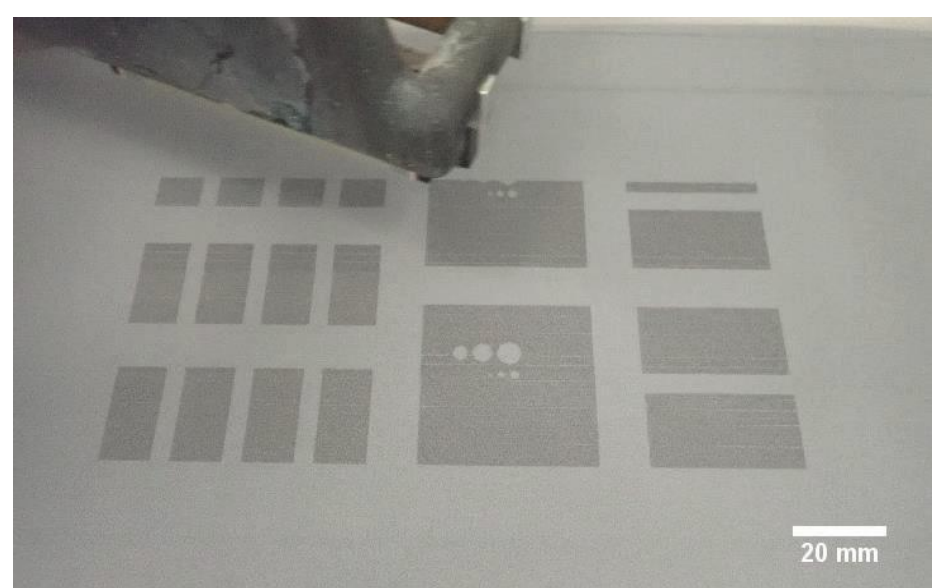

Figure 6: Representative example for the powder bed spreading including binder deposition

Due to the porosity of WC-793 powder, the bulk density is lower than for Amperit powder. The low bulk density results in lots of pores in the sintered part, which causes a low density and poor mechanical properties.

A micrograph of the sintered part is shown in Figure 7. Pores indicate that the sintering process is not yet suitable for producing dense parts. In addition, mixed phases (red marking in Figure 7) were detected. These regions probably belong to the Eta-phase with the phase composition $\mathrm{Co}_{3} \mathrm{~W}_{3} \mathrm{C}$. This phase forms due to a low carbon content, meaning that the residual carbon content from the binder is insufficient to reach an amount of carbon content that does result in Eta-phases. To avoid the Eta-phases, powder with a higher carbon content is to be used for the next tests.

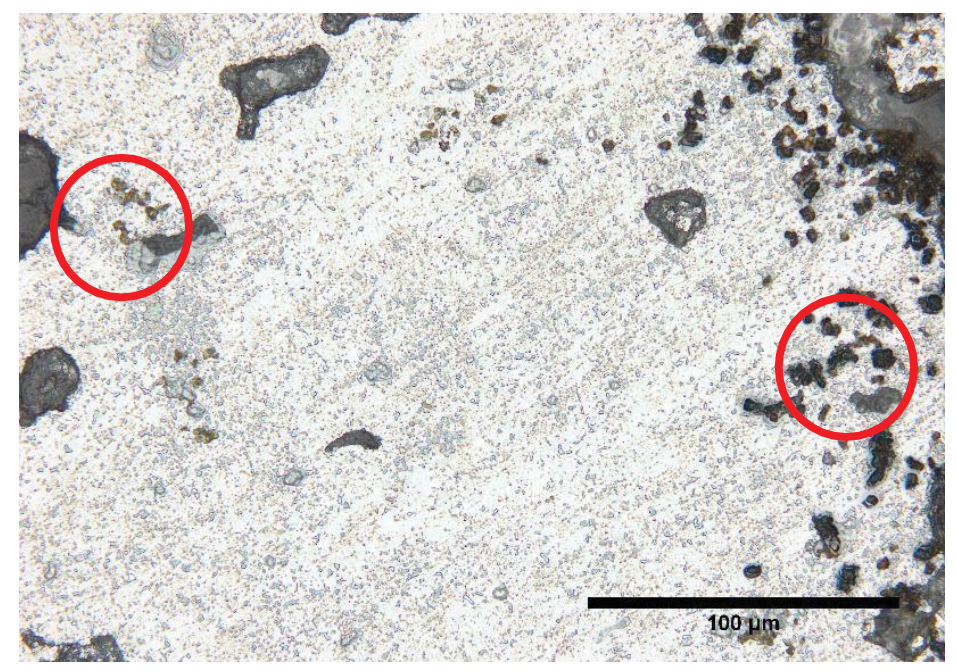

Figure 7: Microscope image of a sintered part

To increase the density after sintering, the nanoparticle-loaded binder is used. The maximum particle size must not exceed $2 \mu \mathrm{m}$. Otherwise clogging might occur in the print head. The particle distributions in the aqueous binders of the three chosen dispersants are shown in Figure 8. The optimum results for each dispersant were taken so that the time in the ultrasonic bath and the concentrations of the dispersants differ. A distribution without dispersant was used as a reference. 


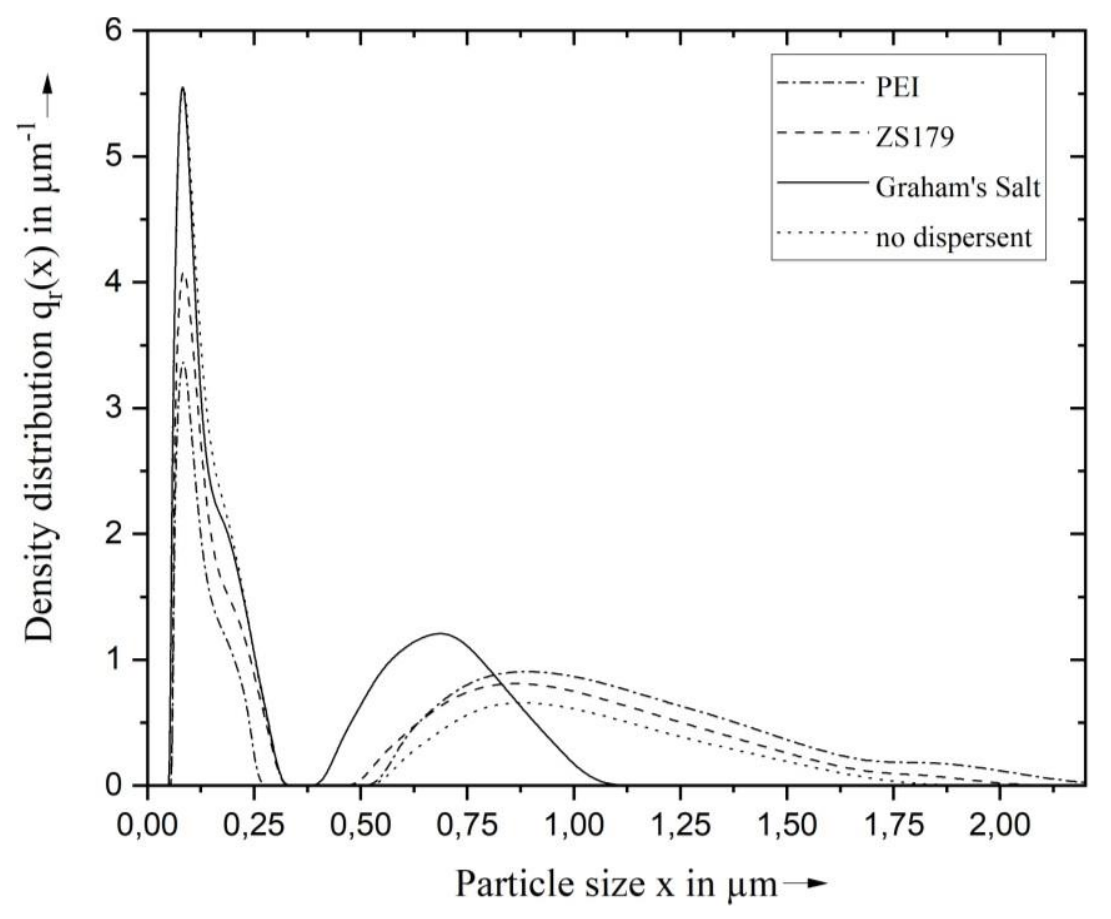

Figure 8: Particle size distribution in the aqueous binder after the use of different dispersants

The density distribution $\mathrm{q}_{\mathrm{r}}(\mathrm{x})$ is plotted against the particle size $\mathrm{x}$. The particle size distribution is bimodal. For all dispersants, the maximum size is smaller than $2.5 \mu \mathrm{m}$. Particle agglomeration was observed for all dispersants. The major fraction of the particles remains in the form of agglomerates with a diameter between 0.5 and $2.5 \mu \mathrm{m}$ with exception of the measurement with Graham's salt. This showed smaller agglomerates with a size between 0.4 and $1.1 \mu \mathrm{m}$. It can be concluded that the dispersants PEI and ZS179 are not suitable for the production of a stable suspension with the aqueous binder. By contrast, Graham's salt shows promising potential. However, further research regarding ultrasonic dwell time, $\mathrm{pH}$, viscosity, and zeta potential is needed to produce a stable binder. Moreover, all parameters for reliable droplet formation of the binder must be tested to ensure its printability.

\section{Conclusion and Outlook}

The presented approach aims at taking functional graded materials to a new level. A theoretical approach for integrating the formation of a cobalt gradient in the fabrication of WC-Co tools and the simultaneous exploitation of the advantages of AM (i.e. BJ) have been presented. The challenges regarding the printing process, gradient formation, gradient propagation, and gradient control have been described. Furthermore, important points for binder production were discussed. BJ represents a suitable process for generating a voxel-based gradient. It is possible to apply particle-loaded binders locally and thereby vary the material compositions. The placement of the particles is controllable through the prediction of the binder saturation.

The presented concept will be analyzed in future investigations. The effect of the input variables on the output variables must be quantified and the influence on the gradient needs to be analyzed. Where the transfer from laboratory to industry is concerned, the feasibility of the process with large systems and several print heads must be considered in respect of scalability and efficiency. Initial 
results show that particle morphology is not crucial to flowability. However, the carbon content of the powder must be taken into account; otherwise unwanted mixing phases will form.

\section{Acknowledgements}

We hereby express our gratitude to the AiF for the funding of this work, within the framework ZIM of the Federal Ministry for Economic Affairs and Energy on the basis of a decision by the German Bundestag, for the funding of our research. Some of the illustrations and results in this paper were achieved within the scope of the research project "ForAdd-HMTools" (grant number 16KN039434)

Supported by:

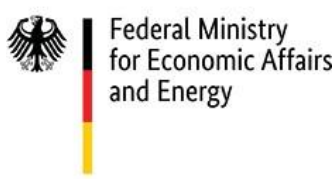

on the basis of a decision by the German Bundestag 


\section{$\underline{\text { References }}$}

[1] R. K. Enneti and K. C. Prough, "Wear properties of sintered WC-12\%Co processed via Binder Jet 3D Printing (BJ3DP)," International Journal of Refractory Metals and Hard Materials, vol. 78, pp. 228-232, 2019.

[2] R. K. Enneti et al., "Sintering of WC-12\%Co processed by binder jet 3D printing (BJ3DP) technology," International Journal of Refractory Metals and Hard Materials, vol. 71, pp. 28-35, 2018.

[3] I. Gibson, D.W. Rosen, B. Stucker, Additive Manufacturing Technologies: 3D Printing, Rapid Prototyping, and Direct Digital manufacturing, 2nd., Springer, New York, (2015), pp. 1-498.

[4] C. Colin et al., "Processing of functional-gradient WC-Co cermets by powder metallurgy," International Journal of Refractory Metals and Hard Materials, vol. 12, no. 3, pp. 145-152, 1993.

[5] G.S. Upadhyaya, "Materials science of cemented carbides - an overview," Materials \& Design, vol. 22, no. 6, pp. 483-489, 2001.

[6] P. Fan, Z. Z. Fang, and J. Guo, "A review of liquid phase migration and methods for fabrication of functionally graded cemented tungsten carbide," International Journal of Refractory Metals and Hard Materials, vol. 36, pp. 2-9, 2018.

[7] A. F. Lisovsky, "The migration of metal melts in sintered composite materials," InInt. J. Heat MassTransfer, no. 33, pp. 1599-1603, 1990.

[8] M. Tokita, "Large-Size-WC/Co Functionally Graded Materials Fabricated by Spark Plasma Sintering (SPS) Method,” MSF, vol. 423-425, pp. 39-44, 2017.

[9] J.-P. Kruth, M. C. Leu, and T. Nakagawa, "Progress in Additive Manufacturing and Rapid Prototyping," CIRP Annals, vol. 47, no. 2, pp. 525-540, 1998.

[10] P. Kunchala and K. Kappagantula, "3D printing high density ceramics using binder jetting with nanoparticle densifiers," Materials \& Design, vol. 155, pp. 443-450, 2018.

[11] A. C. Bailey, M. M. Basti, A. Elliott, and A. Merrima, "Preliminary Testing of Nanoparticle Effectiveness in Binder Jetting Applications,"

[12] D. Godlinski and S. Morvan, "Steel Parts with Tailored Material Gradients by 3D-Printing Using Nano-Particulate Ink,” MSF, vol. 492-493, pp. 679-684, 2016.

[13] Y. Bai, G. Wagner, and C. B. Wiiliams, "Effect of bimodal powder mixture on powder packing density and sintered density in binder jetting of metals," 2018 Annual International Solid Freeform Fabrication Symposium, vol. 62, 2015.

[14] A. Baldan, "Review Progress in Ostwald ripening theories and their applications to nickelbase superalloys: Ostwald ripening theories," Journal of Materials science, vol. 37, pp. 2171-2202, 2017.

[15] B. R. Utela, D. Storti, R. L. Anderson, and M. Ganter, "Development Process for Custom Three-Dimensional Printing (3DP) Material Systems,” J. Manuf. Sci. Eng., vol. 132, no. 1, p. $11008,2018$. 
[16] O. Eso, Z. Z. Fang, and A. Griffo, "Kinetics of cobalt gradient formation during the liquid phase sintering of functionally graded WC-Co," International Journal of Refractory Metals and Hard Materials, vol. 25, no. 4, pp. 286-292, 2007.

[17] A. Formisano et al., "Influence of Eta-Phase on Wear Behavior of WC-Co Carbides," Advances in Tribology, vol. 2016, pp. 1-6, 2016.

[18] Y. Hwang et al., "Production and dispersion stability of nanoparticles in nanofluids," Powder Technology, vol. 186, no. 2, pp. 145-153, 2008.

[19] H. Miyanaji, S. Zhang, and L. Yang, “A new physics-based model for equilibrium saturation determination in binder jetting additive manufacturing process," International Journal of Machine Tools and Manufacture, vol. 124, pp. 1-11, 2018.

[20] B. Utela, D. Storti, R. Anderson, and M. Ganter, "A review of process development steps for new material systems in three dimensional printing (3DP)," Journal of Manufacturing Processes, vol. 10, no. 2, pp. 96-104, 2008.

[21] E. Laarz and L. Bergström, "Dispersing WC-Co powders in aqueous media with polyethylenimine," International Journal of Refractory Metals and Hard Materials, vol. 18, no. 6, pp. 281-286, 2000.

[22] S. Bastian, "Neuro- und Gliotoxizität von Wolframcarbid-basierten Nanopartikeln in vitro," Dissertation, TU Dresden, Dresden, 2018. 